\title{
Handling Analysis and Defining Conditions of Dangerous-Sfe Divergent Stability Loss of a Two-Link Road Train Nonlinear Model
}

\author{
Verbitskii Vladimir Grigorievich, Bezverhyi Anatoliy Igorevich, Tatievskyi Dmitry Nikolayevich* \\ Department of Computerized System Software, Faculty of Power Engineering, Electronics and Information Technologies, Zaporizhia State \\ Engineering Academy, Zaporizhia, Ukraine \\ Email address: \\ oxsidan@ukr.net(V.V. Grigorievich), anatoliy.bezverkhyi@hotmail.com(B. A. Igorevich), dtdissert@gmail.com (T. D. Nikolayevich) \\ ${ }^{*}$ Corresponding author
}

To cite this article:

Verbitskii Vladimir Grigorievich, Bezverhyi Anatoliy Igorevich, Tatievskyi Dmitry Nikolayevich. Handling Analysis and Defining Conditions of Dangerous-Sfe Divergent Stability Loss of a Two-Link Road Train Nonlinear Model. Mathematical Modelling and Applications.

Vol. 3, No. 2, 2018, pp. 31-38. doi: 10.11648/j.mma.20180302.11

Received: March 20, 2018; Accepted: May 2, 2018; Published: May 24, 2018

\begin{abstract}
Road train steady motion mode divergent stability loss compiles with the critical according to A.M. Liapunov case of a single zero root. That said both safe and dangerous stability loss scenarios are possible according to N.N. Bautin. Dangerous stability loss is followed with a semi-trailer intensive drifting even in case of linear motion. Analyzing the reasons of such system behavior requires developing new effective analytical approaches towards defining safe-dangerous articulated vehicle divergent stability loss because direct methods for finding corresponding Liapunov indexes may appear ineffective in the analytical form being excessively cumbersome. The work presents a formalized approach to analyzing safe stability loss conditions the essence of which is in defining conditions when bifurcation set structure rearrangement occurs in linear motion critical speed small neighborhood. The kind of approach has been tested by the authors when analyzing single unit vehicle stability. Analytical relations are presented defining road train configuration following circular paths with constant Ackermann angle; consideration of analytical results accuracy evaluation is performed based on comparing to the results received with numerical analytic parameter continuation method; analytical relations are received corresponding to safe linear motion mode stability loss (in the sense of N.N. Bautin). The work develops methods of analyzing two-link vehicle non-linear model two-parameter steady modes manifold stability.
\end{abstract}

Keywords: Divergent Instability, Handling, Steady Mode, Two-Link Road Train

\section{Introduction}

Road train handling properties can differ from the corresponding properties of a separately taken tractor truck to a great extent because of vertical reactions redistribution on the tractor truck centre shafts and transverse reaction appearing in the point of coupling with a semitrailer [1-5]. Special attention from the point of view of traffic security should be paid to analyzing conditions which may cause abrupt changes (folding bifurcation corresponding to the divergent circular steady mode stability loss) semitrailer orientation (internal-external) in the circular mode with varying movement traveling speed [6]. The multitude of parameters when the kind of abrupt transferrings of steady states occur is a critical multitude or a bifurcation multitude with cuspidal points playing significant role in its structure [7, $8,9]$. Typical and the simplest bifurcation set implementation is semicubical parabola though the algorithms of building bifurcation sets in an analytical form even for a case of single vehicle have been missing. The results of Troger H., Zeman K. and Fabio Della Rossaa $[10,11]$ were based on a numeric two parameter continuation method $[12,13]$, this complicates defining safe conditions of linear movement mode stability loss in a space of parameters according to N.N. Bautin [14]. In the works of L.G. Lobas, V.G. Verbitskii $[6,15-17]$ the questions of qualitative analyses of wheel vehicles non-linear models have been reviewed involving bifurcation theory elements. A similar approach is implemented in the present 
work allowing to receive a more complete bolster - type road train model bifurcation multitude representation in an analytical form. The work is inspired by J.P. Pauwelussen [18] results and is aimed at further development of graph-analytical approach to analyzing two-link road train nonlinear model steady states multitude using the idea of bifurcation analyses.

\section{Problem State}

Task setting. A critical controlling parameters multitude should be found $\theta, v$ responsible for two-link road train steady states multitude divergent stability loss. Conditions for steady circular states divergent stability loss should be defined which correspond to movement with varying traveling speed along a curve of a set bend radius (with a fixed Ackermann angle).

Task solving method. A geometric method of analyzing a road train nonlinear model steady states multitude [18] combined with dynamic systems bifurcations theory approaches [8] give a possibility to get divergent stability loss conditions on a handling curve in a general form (a condition of divisible steady states implementation).

A number of simplifying suggestions are accepted in the work traditional to tasks of graph-analytical analyses of steady states variety for bolster-type road train bicycle mode [18, 19]. A scheme of an articulate vehicle is given on figure 1, here $v-$ tractor truck centre line of mass direct-axis component; $\theta-$ controlled module turning angle; $\mathrm{a}, \mathrm{b}-\mathrm{a}$ distance from tractor truck centre line of mass to tractor truck front (controlled) axle and rear axle centres; $\mathrm{c}-\mathrm{a}$ distance from tractor track centre line of mass to coupling with the second link; $d_{1}-$ a distance from the second link centre line of mass to the point of coupling with a tractor truck; $Y_{i}-$ vehicle drift force on axles, resistance coefficients to vehicle drift on axles $\left(k_{1} ; k_{2} ; k_{3}\right) ; \mathrm{m}$ tractor truck mass; $\mathrm{u}$ - transverse tractor truck centre line of mass velocity vector projection; $\omega$ - tractor truck rotational speed in correspondence to vertical axis; $\mathrm{m}_{2}$ - the second link mass; $\mathrm{v}_{1}, \mathrm{u}_{1}-$ lengthwise and transverse semitrailer centre line of mass velocity vector projection; $\varphi$ - folding angle (the angle between the tractor truck and semitrailer rolling axis).

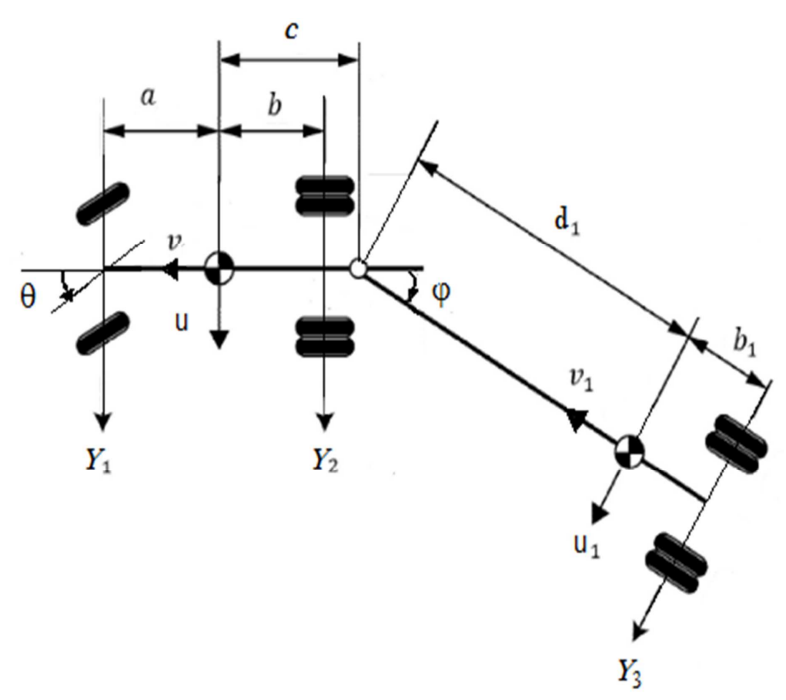

Figure 1. A two-link road train structural scheme.

\section{Analytical Representation of a Handling Curve (a Nonlinear Approach)}

The source system defining two-link road train nonlinear model steady states multitude has the looks of:

$$
\begin{gathered}
-\left(m+m_{2}\right) \omega v+Y_{1}+Y_{2}+Y_{3} \cos \phi+m_{2} d_{1} \omega^{2} \sin \phi=0 ; \\
c m_{2} \omega v+a Y_{1}-b Y_{2}-c Y_{3} \cos \phi-c m_{2} d_{1} \omega^{2} \sin \phi=0 ; \\
L_{1} Y_{3}-m_{2} d_{1} \omega v \cos \phi+m_{2} d_{1} \omega u \sin \phi-c m_{2} d_{1} \omega^{2} \sin \phi=0 ; \\
\delta_{1}=\theta-\frac{u+a \omega}{v} ; \\
\delta_{2}=\frac{-u+b \omega}{v} ; \\
\delta_{3}=\frac{-u_{1}+b_{1} \omega}{v_{1}} ; \\
u_{1}=(u-c \omega) \cos \phi+v \sin \phi-d_{1} \omega ; \\
v_{1}=v \cos \phi-(u-c \omega) \sin \phi .
\end{gathered}
$$

A simplified (partially linear) equations system defining road train movement steady mode:

$$
\begin{gathered}
-\left(m+m_{2}\right) \omega v+Y_{1}+Y_{2}+Y_{3}=0 ; \\
c m_{2} \omega v+a Y_{1}-b Y_{2}-c Y_{3}=0 ; \\
-d_{1} m_{2} \omega v+L_{1} Y_{3}=0,
\end{gathered}
$$

can be solved in relation to drift forces implemented on axles:

$$
\bar{Y}_{1}\left(\delta_{1}\right)=\bar{Y}_{2}\left(\delta_{2}\right)=\bar{Y}_{3}\left(\delta_{3}\right)=\frac{v^{2}}{g R},
$$

where $\bar{Y}_{i}=Y_{i} / N_{i}-$ a dimensionless drift force on i-n axle [18]; linear drift angles:

$$
\begin{aligned}
& \delta_{1}=\theta-\frac{u+a \omega}{v} ; \delta_{2}=\frac{-u+b \omega}{v} ; \\
& \delta_{3}=-\phi+\frac{-u+\left(L_{1}+c\right) \omega}{v}
\end{aligned}
$$

vertical reactions on axles:

$$
\begin{aligned}
& N_{1}=\frac{b L_{1} m-(c-b) b_{1} m_{2}}{L_{1} l} ; \\
& N_{2}=\frac{(a+c) b_{1} m_{2}+a L_{1} m}{L_{1} l} ; \\
& N_{3}=\frac{m_{2} d_{1}}{L_{1}} .
\end{aligned}
$$


With a set movement along the circle with a set radius the $\omega=\frac{v}{R}$ correlation is present where $\mathrm{v}$ - direct-axis tractor truck centre line of mass (C.M.) component, $R-$ dot locus radius on a tractor truck rolling axis the speed of which is directed along the tractor truck rolling axis.

Taking into account that the difference of drift angles on the first and second tractor truck axes $\delta_{1}-\delta_{2}=\theta-l / R$, on the third and the second axes $\delta_{3}-\delta_{2}=-\phi+\left(L_{1}+c-b\right) / R$, and drift angles values corresponding to movement steady states $\delta_{\mathrm{i}}: \quad \delta_{1}=\bar{Y}_{1}^{-1}\left(\bar{a}_{y}\right), \delta_{2}=\bar{Y}_{2}^{-1}\left(\bar{a}_{y}\right), \delta_{3}=\bar{Y}_{3}^{-1}\left(\bar{a}_{y}\right)$, two correlations are received defining handling curve and folding curve in case of an articulated vehicle:

$$
\begin{gathered}
\theta=l / R+\bar{Y}_{1}^{-1}\left(\bar{a}_{y}\right)-\bar{Y}_{2}^{-1}\left(\bar{a}_{y}\right) ; \\
-\phi=-\left(L_{1}+c-b\right) / R+\bar{Y}_{3}^{-1}\left(\bar{a}_{y}\right)-\bar{Y}_{2}^{-1}\left(\bar{a}_{y}\right),
\end{gathered}
$$

where $\overline{\mathrm{a}}_{\mathrm{y}}=v^{2} /(R g)-$ transverse tractor truck C.M. acceleration component.

The system (3) can be presented in a more general form:

$$
\begin{aligned}
\theta= & l / R+\bar{Y}_{1}^{-1}\left(\bar{a}_{y}\right)-\bar{Y}_{2}^{-1}\left(\bar{a}_{y}\right)=l \cdot g \cdot \bar{a}_{y} / v^{2}+G_{1}\left(\bar{a}_{y}\right)-G_{2}\left(\bar{a}_{y}\right) \\
=l \cdot g & \cdot \bar{a}_{y} / v^{2}+G_{12}\left(\bar{a}_{y}\right) \\
& \quad-\phi=-\left(L_{1}+c-b\right) / R+\bar{Y}_{3}^{-1}\left(\bar{a}_{y}\right)-\bar{Y}_{2}^{-1}\left(\bar{a}_{y}\right) \\
& =-\left(L_{1}+c-b\right) \cdot g \cdot \bar{a}_{y} / v^{2}+G_{32}\left(\bar{a}_{y}\right)
\end{aligned}
$$

allowing to define phase variables values corresponding to a steady mode with set controlling parameters values $\theta, v$.

Thus, from the first system equation (4) it can be concluded that steady states parameters of which are going to depend on corresponding $\bar{a}_{y}=v^{2} /(R \cdot g)=v \cdot \omega / g$ argument values correspond to the cross points of "movable" right line $l \cdot g \cdot \bar{a}_{y} / v^{2}-\theta$ and "fixed" curve $G_{21}\left(\bar{a}_{y}\right)=-G_{12}\left(\bar{a}_{y}\right)$.

The corresponding folding angle value is received from the second equation (4). This way the simplified equation system (2) leads to splitting the source system (1). In this case the first system equation (4) is defining from the point of view of divergent stability loss conditions (has the same form as in case of a separately taken tractor track).

A criterion of steady states stability found from the first defining system equation (4) based on a geometric interpretation can be summarized as follows: a steady mode is stable if the inclination of movable right line is larger than the inclination of fixed curve in the corresponding crossing point (figure 1). A divisible steady mode is going to correspond to divergent stability loss, i.e. the contact of movable right line $l \cdot g \cdot \overline{\mathrm{a}}_{\mathrm{y}} / v^{2}-\theta$ and fixed curve $G_{21}\left(\bar{a}_{y}\right)$, which leads to the following correlation for controlling parameters:

$$
\begin{aligned}
& \theta=\bar{a}_{y} \cdot G_{21}^{\prime}\left(\bar{a}_{y}\right)-G_{21}\left(\bar{a}_{y}\right) ; \\
& v=\left(l \cdot g / G_{21}^{\prime}\left(\bar{a}_{y}\right)\right)^{1 / 2}
\end{aligned}
$$

The conditions when systems (1) and (2) have quantitative discrepancies only should be analyzed. The numerical analytical parameter continuation method should be used for building balanced curves corresponding to the source system (1). Balanced curves corresponding to system (2) can be set analytically in a parameters form (according to rotational speed):

$$
\begin{aligned}
& \theta=l \cdot g \cdot \bar{a}_{y} / v^{2}+G_{12}\left(\bar{a}_{y}\right) ; \\
& \omega=\bar{a}_{y} \cdot g / v
\end{aligned}
$$

and (according to folding angle):

$$
\begin{aligned}
& \theta=l \cdot g \cdot \bar{a}_{y} / v^{2}+G_{12}\left(\bar{a}_{y}\right) ; \\
& -\phi=-\left(L_{1}+c-b\right) \cdot g \cdot \bar{a}_{y} / v^{2}+G_{32}\left(\bar{a}_{y}\right) .
\end{aligned}
$$

Balanced system (1) and (2) curves are represented on figure 2 received for the following parameters numeric values $\mathrm{g}=9,81 \mathrm{~m} / \mathrm{s}^{2} ; \mathrm{a}=4,217 \mathrm{~m} ; \mathrm{b}=3,376 \mathrm{~m} ; \mathrm{b}_{1}=2,93 \mathrm{~m} ; \mathrm{d}_{1}=$ $8,075 \mathrm{~m} ; \mathrm{c}=3,376 \mathrm{~m} ; \mathrm{m}=6417 \mathrm{~kg} ; \mathrm{m}_{2}=41846 \mathrm{~kg}$; $\mathrm{k}_{1}=100000 \mathrm{H} ; \mathrm{k}_{2}=300000 \mathrm{H} ; \mathrm{k}_{3}=300000 \mathrm{H} ; \kappa_{1}=0,8 ; \kappa_{2}=0,8$; $\kappa_{3}=0,8$; axial forces in the contact area of wheels with bearing surface haven't been taken into account; rotational drift forces were take approximate as an arctangensoid:

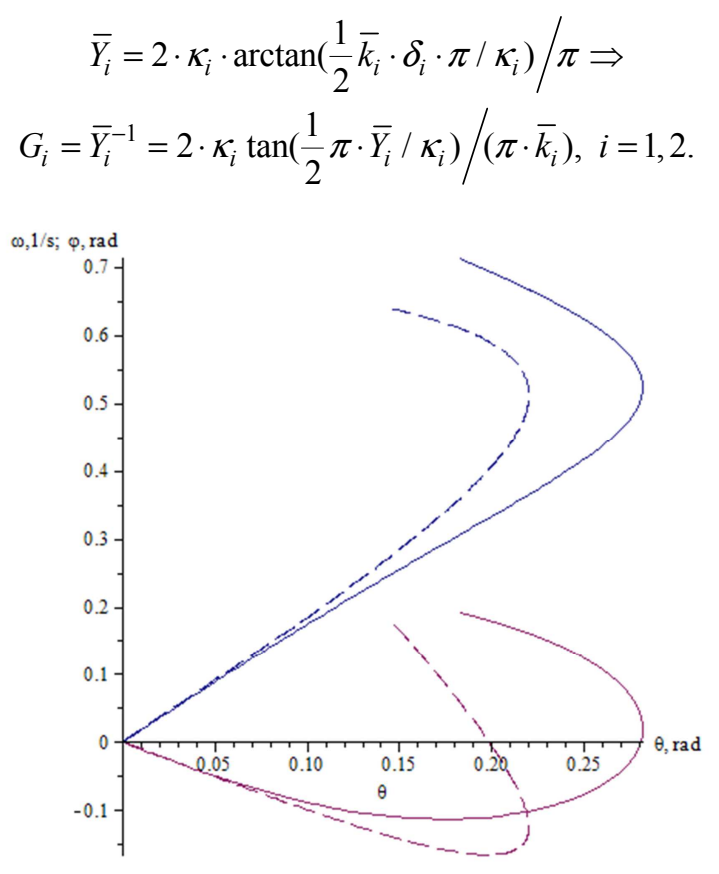

Figure 2. Balanced curves at a speed of $10 \mathrm{~m} / \mathrm{s}$.

$\omega(\theta)$ - the navy colour, the solid curve for system (1), the dashed one for (2); $\varphi$ $(\theta)$ - the maroon colour, the solid curve for system (1), the dashed one for system (2)

It should be noted that steady mode divergent stability loss corresponds to the turning points on the balanced curves with a corresponding controlling angle value $\theta$. Thus, the source system (1) critical parameters multitude is a bit different from the system (2) critical multitude, besides with sufficiently small 
movement traveling speed (in comparison with the critical linear motion speed) there are quality difference there - there is a traveling speed interval there for the source system for which divergent stability loss is not implemented as opposed to the simplified system (2). Nevertheless, the analyses of system (2) divergent stability loss can be quite useful at the first stage of researching the results of which can be made more specific as required base on the complete system (1).

\subsection{Divergent Stability Loss Criterion}

Analyzing steady circular states divergent stability loss satisfying the handling curve (the first system (3) equation). As the reviewed case is a particular case of losing steady circular mode stability, this gives a possibility to define common points (if they exist) when combining stability diagram (bifurcation multitude) and handling curve on the same coordinate plane.

The condition of common points presence between a handling curve and a bifurcation multitude can be written down in an analytical form:

$$
G_{21}^{\prime}\left(\bar{a}_{y}\right) \cdot \bar{a}_{y}=l / R
$$

It can be concluded from the "geometric" stability criterion that the steady circular states divergent stability loss corresponding to the handling curve is related to breaking the inequation:

$$
G_{21}^{\prime}\left(\bar{a}_{y}\right) \cdot \bar{a}_{y}<l / R
$$

Let the handling curve be equal to the $\mathrm{R}=30,5 \mathrm{~m}$ parameter solving equation (6) in relation to $\overline{\mathrm{a}}_{\mathrm{y}}$, the following is received $\overline{\mathrm{a}}_{\mathrm{y}}=0,52717$, putting this value of scaled transverse acceleration into the handling equation gives a critical parameter value $\theta=0,1081 \mathrm{rad}$, a critical parameter value $v=$ $12,559 \mathrm{~m} / \mathrm{s}$ can be found from the correlation $\bar{a}_{y}=v^{2} /(R \cdot g)$.

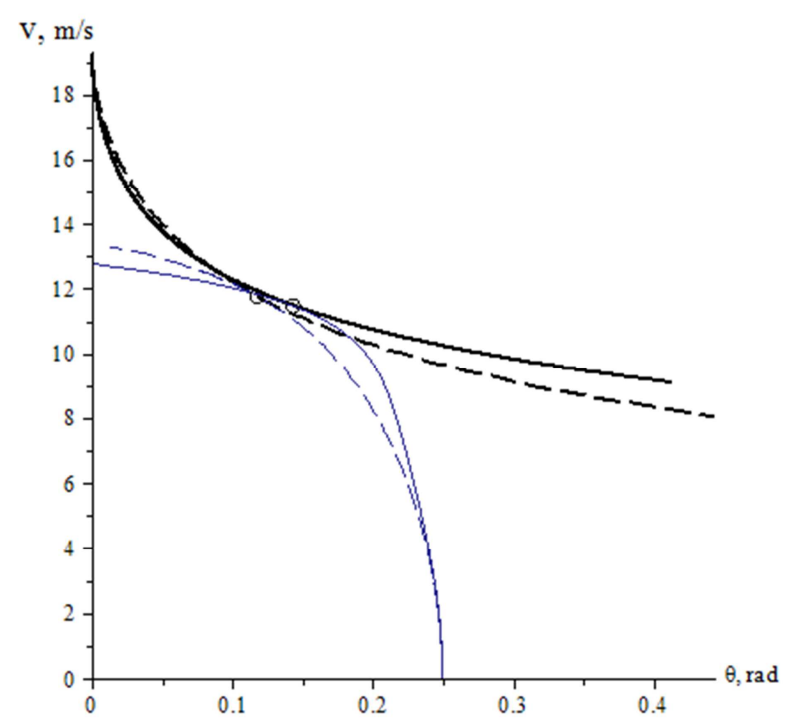

Figure 3. Defining controlling parameters critical values corresponding to divergent stability loss on handling curve using bifurcation diagram (solid curves correspond to system (1), dashed curves to system (2)).
Divergent instability on handling curve for the source system (1) implements with the speed of $v=11,5 \mathrm{~m} / \mathrm{s}-$ it's received based on the numerical analytical two parameter continuation method.

In case of the simplified system (2) geometric rendering of the corresponding steady mode divergent stability loss conditions is possible - divisible mode implementation (figure 3) which is useful when analyzing the results of numerical complete system (1) integration.

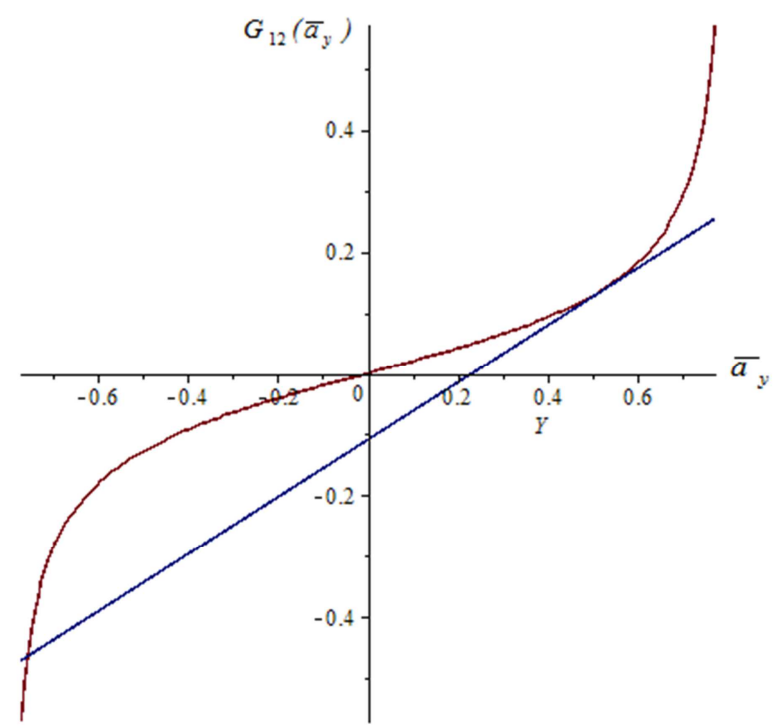

Figure 4. An illustration to a graph-analytical technique for finding steady states corresponding to the set Ackermann angle value; a case of divergent stability loss.

A comparison is available further on for approximate analytical expressions defining handling curve and folding curve with the results of building these curves base on numerical analytical parameter continuation method. The diagrams of the handling curve (the navy colour) and the "folding" curve (the maroon colour) correspond to the flection radius of $\mathrm{R}=30,5 \mathrm{~m}$ (figure 4 ).

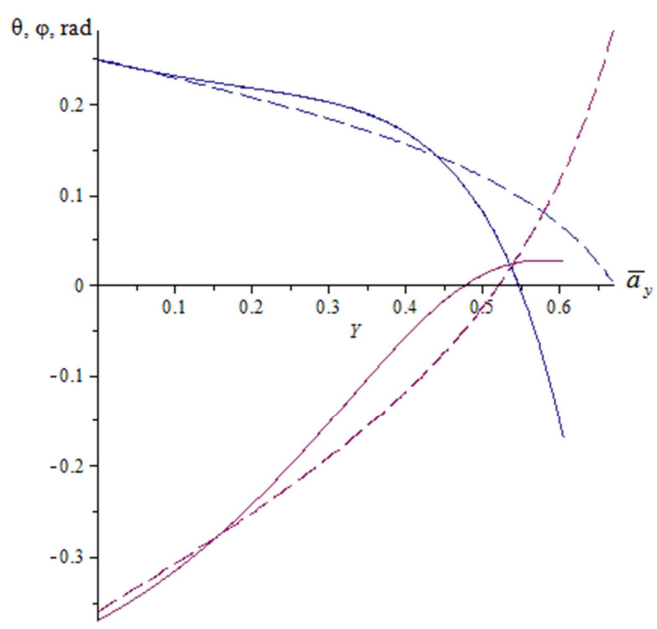

Figure 5. Combining of handling curve and folding curve of a source two-link road train nonlinear model (solid curves) with their approximate variants (dashed curves). 
This way, handling curves and the folding ones (which are received when putting $\bar{a}_{y}=v^{2} /(R \cdot g)$ into (4) can serve as a nonlinear generalization of handling and folding right lines (the later ones correspond to the case of drift forces linear approximation) [20]: the speed of angle folding is defined by the $\frac{N 3}{k 3}-\frac{N 2}{k 2}$ expression (similar to the $\frac{N 1}{k 1}-\frac{N 2}{k 2}$ understeering gradient), the folding angle value at a considerably low speed $v$ is set with $-\frac{L 1+c-b}{R}$ correlation similar to the Ackermann angle $\frac{l}{R}$. And the divergent stability loss (7) condition received when working corrects the stability boarder obtained based on a linear model in a parameters space to a large extent.

\subsection{Conditions of Safe Two-Link Road Train Linear Movement Mode Stability Loss}

The conditions for rebuilding bifurcation multitude structure in small neighbourhood of linear movement critical speed should be defined. To solve the task the source system (1) with the precision up to the third term is going to be brought together to a defining equation (the third extent polynom) in relation to one phase variable.

Let's represent system (1) in an approximate form:

$$
\begin{gathered}
-m \omega v+\bar{Y}_{1} N_{1}+\bar{Y}_{2} N_{2}+Y_{o}=0 ; \\
a \bar{Y}_{1} N_{1}-b \bar{Y}_{2} N_{2}-c Y_{o}=0 ; \\
Y_{o}=\frac{m_{2} \omega\left\{d_{1} \phi\left[-u+\left(L_{1}+c\right) \omega-v \phi\right]-b_{1} v\right\}}{L_{1}} ; \\
\bar{Y}_{3}=\frac{\omega\left[(-u+c \omega) \phi+v\left(1-\phi^{2} / 2\right)\right]}{g},
\end{gathered}
$$

where $Y_{o}$ - transverse reaction in the joint point influencing a tractor track ( $Y_{o}$ and $\bar{Y}_{3}$ are defined with the precision to the third term smallness values). Solving this system in relation to non-dimesional drift forces:

$$
\bar{Y}_{1}=\frac{-b m \omega+Y_{o}(b-c)}{l N_{1}} ; \bar{Y}_{2}=\frac{-a m \omega+Y_{o}(a+c)}{l N_{2}},
$$

switching to inverse functions $\delta_{1}=\bar{Y}_{1}^{-1}\left(x_{1}\right), \delta_{2}=\bar{Y}_{2}^{-1}\left(x_{2}\right)$, $\delta_{3}=\bar{Y}_{3}^{-1}\left(x_{3}\right)$. Then arguments are put into $x_{i}$ dependences:

$$
\begin{gathered}
u=\frac{-\omega v^{2}}{\bar{k}_{2} g}+b \omega ; \\
\phi=\frac{\left(L_{1}-b+c\right) \omega}{v}+\frac{\left(1 / \bar{k}_{2}-1 / \bar{k}_{3}\right) \omega v}{g},
\end{gathered}
$$

correct in linear approximation (after this procedure $x_{i}$ arguments are still going to be set with the precision up to the third term of smallness). Taking into account that in a curve of flection fixed radius $\omega=v / R$, and the difference of drift angles on the first and second tractor truck axels $\delta_{1}-\delta_{2}=\theta-l / R$, on the third and second axels $\delta_{3}-\delta_{2}=-\varphi+\left(L_{1}+c-b\right) / R$, specified to the third term of smallness equations of handling and folding are received similar to equations (3):

$$
\begin{gathered}
\theta=l / R+\bar{Y}_{1}^{-1}\left(x_{1}\right)-\bar{Y}_{2}^{-1}\left(x_{2}\right) \\
-\phi=-\left(L_{1}+c-b\right) / R+\bar{Y}_{3}^{-1}\left(x_{1}\right)-\bar{Y}_{2}^{-1}\left(x_{2}\right)
\end{gathered}
$$

where $x_{1}=x_{1}(v), x_{2}=x_{2}(v)$.

Figure $6(\mathrm{a}, \mathrm{b})$ represents handling and folding curves for comparison corresponding to approximate dependences (3), (8) and curves received based on numerical parameter continuation method for a complete system (1).

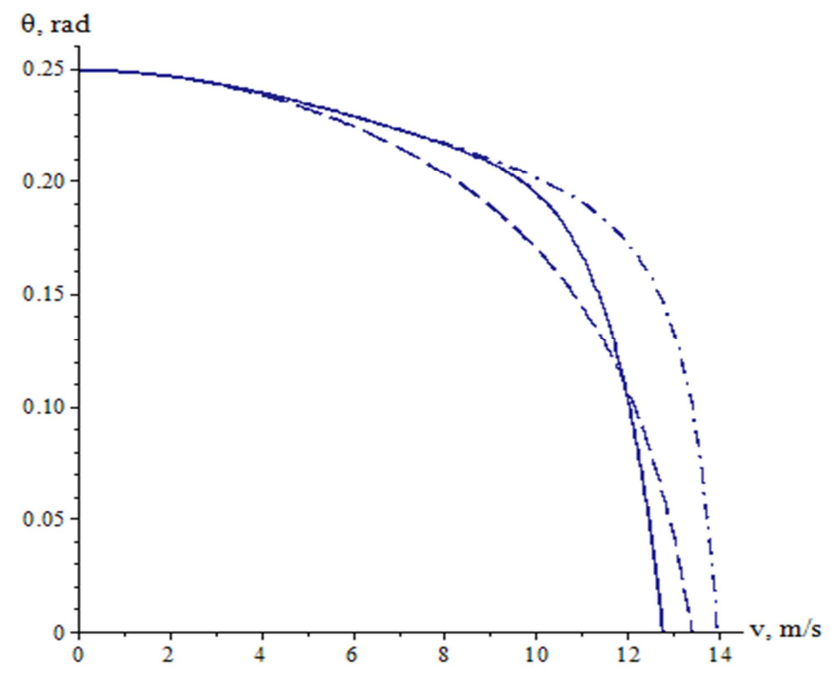

Figure 6. a) handling curve; b) folding curves (the solid curve corresponds to system (1), system (8) - the dash-dotted one, system (3) - the dashed one).

The first system (8) equation without a suggestion of circular locus flection permanence:

$$
\theta=l \cdot \omega / v+\bar{Y}_{1}^{-1}\left(x_{1}\right)-\bar{Y}_{2}^{-1}\left(x_{2}\right), x_{1}=x_{1}(\omega), x_{2}=x_{2}(\omega)
$$

is a defining equation (received with the precision of up to the third term of smallness) from one phase variable rotational speed. With fixed controlling parameters $\theta, v$ it defines steady states multitude. If limiting only with its linear and cubic terms in Taylor series expansion of $\omega$ grades a required polynomial is received. A formalized approach to analyzing safe linear movement mode stability loss is given below. Let's introduce a new generalized $\bar{K}_{i}$ parameter which value is going to depend on drift forces approximation kind:

$$
\delta_{i}=\overline{Y_{i}^{-1}}\left(x_{i}\right)=x_{i} / \bar{k}_{i}+x_{i}^{3} / \bar{K}_{i}, i=1,2 .
$$

Further result is going to be received for a particular case of $c=b, b_{I}=0$, in this case coefficients of the defining polynomial 
can be represented in a visible form:

$$
0=-\theta+\frac{l}{v}\left(1-v^{2} / V_{1}^{2}\right) \omega+\frac{v}{g^{3}}\left[v^{2}\left(1 / \bar{K}_{1}-1 / \bar{K}_{2}\right)+d_{1} \cdot g \cdot\left(m_{1} / m\right) \cdot(1+b / a) \cdot\left(1-v^{2} / V_{2}^{2}\right) /\left(\bar{k}_{2} \cdot \bar{k}_{3}\right)\right] \omega^{3}
$$

From analyzing coefficients signs for linear and cubic terms in small neighbourhood of critical velocity $\mathrm{v}<\mathrm{V}_{1}$ and $\mathrm{v}>\mathrm{V}_{1}$ it can be concluded that safe linear movement mode stability loss $(\theta=0)$ implements for a positive coefficient value with the highest term of the defining polynomial $(\mathrm{v}=\mathrm{V} 1$ should be considered in the later one) - in this case with the supercritical velocity a pair of steady circular movement states appears limiting the unstable linear mode destabilization growth. Then at least within the small neighbourhood of linear movement mode critical velocity, the boarder of bifurcation multitude should be higher that the cuspidal point (cusp) indicating the implementation of threefold movement mode with $v=V_{l}, \theta$ $=0$. The mentioned condition is equivalent to the condition of linear movement mode stability in a critical case of zero root (in the A.M. Lyapunov [21] stability theory), though this approach leads to considerable simplification of concurrent calculations.

In other words, bifurcation multitude in a small neighbourhood of linear movement critical velocity co-insides with a defining polynomial discriminant curve. Earlier in the work [22] analytical correlations were received corresponding to the conditions of safe linear movement mode stability loss for parameters which considerably influence rebuilding of bifurcation mode relating to $d_{1}, b_{1}, m_{2}, k_{3}$. Though it's necessary to remember that results strongly depend on a particular approximation kind of drift forces dependences. Thus in the present work drift forces have been approximated as an arctangensoid, in the work [22] fractional surdic approximation was used which with other equal conditions led to discrepancy of the received critical $d_{1}$ parameter value at about $5 \mathrm{~m}$. Above is the condition of safe two section road train linear movement mode stability loss which in a certain sense doesn't depend on a particular drift forces approximation kind - the chosen drift forces approximation type should allow transferring to inverse functions or at last allow the possibility of defining their Taylor series expansion first nonlinear (cubic) terms:

$$
\begin{aligned}
& \delta_{i}=\bar{Y}_{i}^{-1}\left(x_{i}\right)=x_{i} / \bar{k}_{i}+x_{i}^{3} / \bar{K}_{i}, i=1,2 . \\
& V_{1}^{2}\left(1 / \bar{K}_{1}-1 / \bar{K}_{2}\right)+d_{1} \cdot g \cdot\left(m_{1} / m\right) \cdot(1+b / a) \cdot\left(1-V_{1}^{2}\right) /\left(\bar{k}_{2} \cdot \bar{k}_{3}\right)>0,
\end{aligned}
$$

where:

$$
\begin{aligned}
& \bar{V}_{1}^{2}=\bar{k}_{1} \cdot \bar{k}_{2} l \cdot g /\left(\overline{k_{1}}-\overline{k_{2}}\right) ; \\
& \bar{V}_{2}^{2}=\bar{k} 2 \cdot \overline{k_{3}} d_{1} \cdot g /\left(\overline{k_{3}}-\overline{k_{2}}\right) ; \\
& 1 / \bar{K}_{i}=\left(\pi^{2} / 12\right) /\left(\overline{k_{i}}-\overline{k_{i}^{2}}\right), i=1,2
\end{aligned}
$$

in case of drift forces approximation as an arctangensoid.

The addend in inequation (9) is generated by linear approximation terms and this makes it stable with various kinds of wheel slipping forces approximation. $V_{l}$ value defines critical velocity of an articulate vehicle linear movement mode, $V_{2}-$ is a value possessing dimension of velocity.

Figure 7, (a, c) depicts the influence of peculiar $d_{1}$ parameter variation in its critical value neighbourhood $\left(\mathrm{d}_{1}{ }^{*}\right.$ $=14,435 \mathrm{~m}$ ) on bifurcation multitude type, bifurcation curves are build for undercritical $\left(d_{1}=14 \mathrm{~m}\right)$ and supercritical $\left(\mathrm{d}_{1}=15\right.$ $\mathrm{m})$ parameter values, bifurcation curves fragments in small neighbourhood of linear movement mode critical velocity are represented on figure $7(\mathrm{~b}, \mathrm{~d})$. Qualitative changes in bifurcation diagram structure (the later are received numerically based on the two parameter continuation method) correlate with the analytical result (9). With numerical building the following set of parameters was used $\mathrm{g}=9,81$ $\mathrm{m} / \mathrm{s}^{2} ; \mathrm{a}=1,92 \mathrm{~m} ; \mathrm{b}=0,82 \mathrm{~m} ; \mathrm{c}=0,82 \mathrm{~m} ; \mathrm{m}=5310 \mathrm{~kg} ; \mathrm{m}_{2}=$ $6481 \mathrm{~kg} ; \mathrm{k}_{1}=305091 \mathrm{H} ; \mathrm{k}_{2}=103496 \mathrm{H} ; \mathrm{k}_{3}=154079 \mathrm{H} ; \kappa_{1}=0,8$; $\kappa_{2}=0,8 ; \kappa_{3}=0,8$.

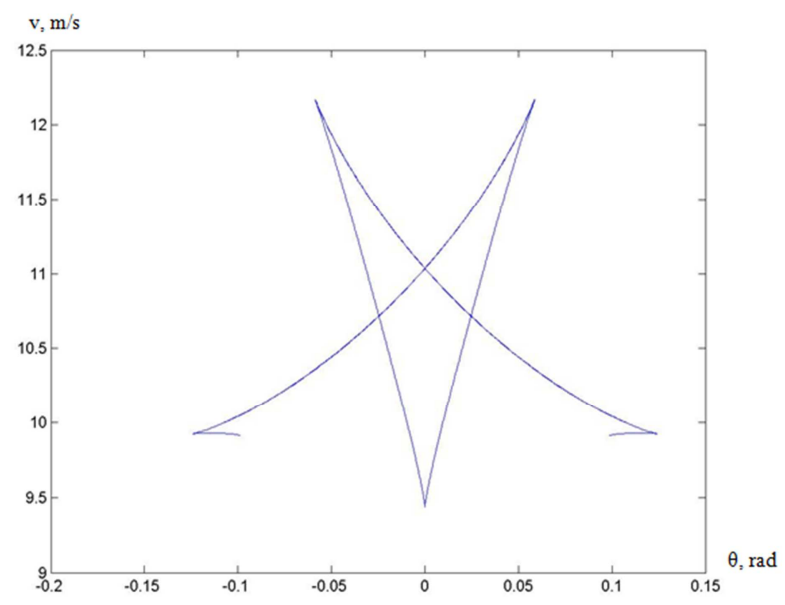

a)

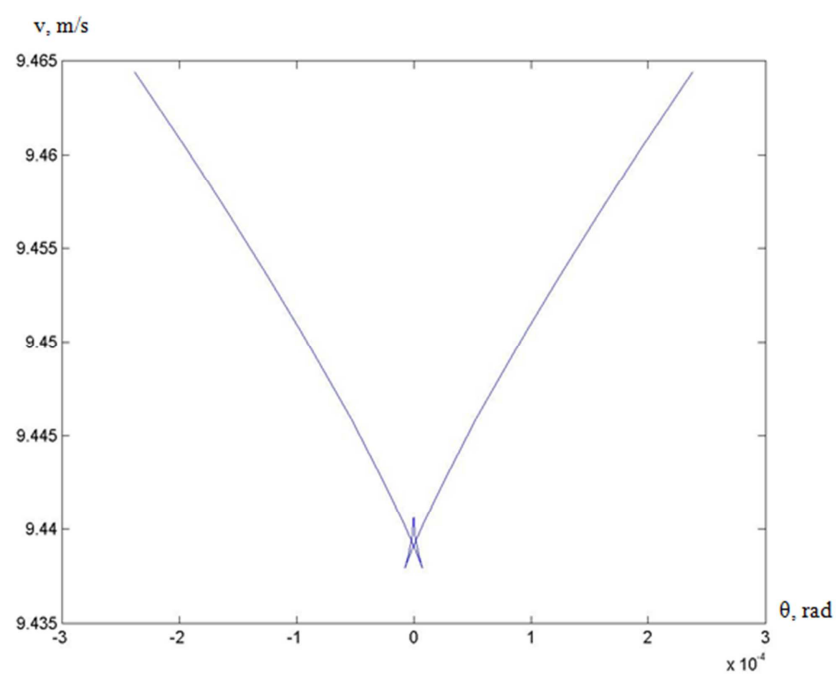

b) 


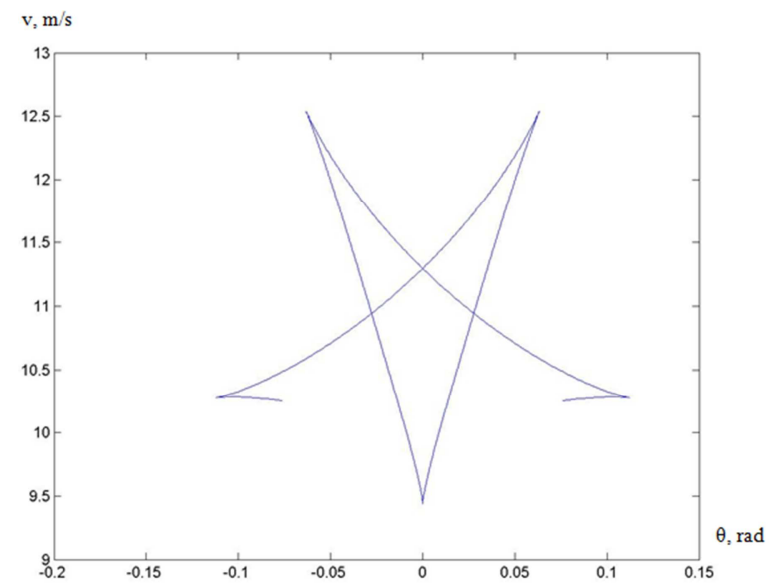

c)

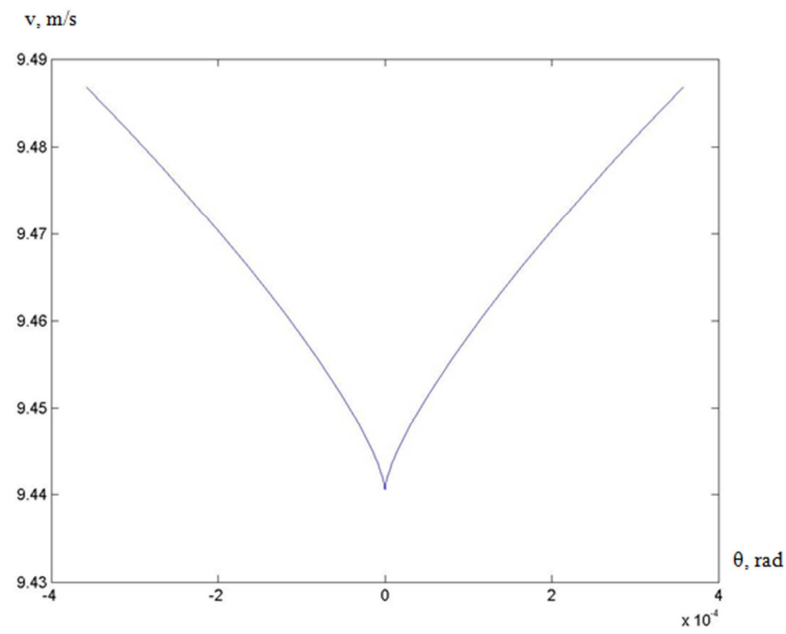

d)

Figure 7. Controlling parameters bifurcation multitude corresponding to system (1).

a) bifurcation multitude with undercritical $d_{l}$ parameter value; b)- bifurcation multitude fragment with undercritical $d_{l}$ parameter value in central cusp neighbourhood; c) bifurcation multitude with supercritical $d_{l}$ parameter value; d) bifurcation multitude fragment with supercritical $d_{l}$ parameter value in central cusp neighbourhood

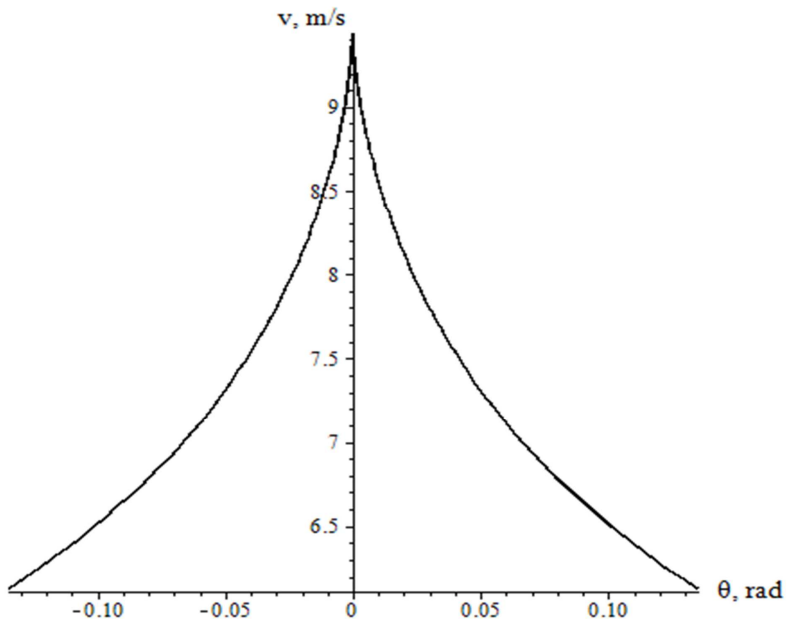

Figure 8. Bifurcation multitude of controlling parameters with supercritical $d_{1}$, parameter value corresponding to system (2).
Bifurcation multitude of complete system (1) and simplified system (2) can have considerable differences - this comes out of the safe linear movement mode stability loss conditions received analytically. Thus figure 8 represents bifurcation multitude corresponding to approximate system (2) to compare it to its exact equivalent on figure 7 (c).

For system (2) conditions of safe stability loss can be easily obtained from the corresponding defining equation (the first (4) equation) represented with the precision of up to cubic terms of scaled transverse acceleration power expansion:

$$
\begin{aligned}
& \theta=l \cdot g \cdot \bar{a}_{y} / v^{2}+G_{12}\left(\bar{a}_{y}\right)=> \\
& =\theta+\left(g l / v^{2}+1 / \bar{k}_{1}-1 / \bar{k}_{2}\right) \bar{a}_{y}+\left(1+\bar{K}_{1}-1 / \bar{K}_{2}\right) \bar{a}_{y}^{3}=0 . \\
& 1 / \bar{K}_{i}=\left(\pi^{2} / 12\right) /\left(\bar{k}_{i} \cdot \bar{k}_{i}^{2}\right), i=1,2 .
\end{aligned}
$$

Analyzing solutions number of the latest equation in small critical velocity neighbourhood $v=V_{1}$ (it's supposed that $\left.\bar{k}_{1}>\overline{\mathrm{k}}_{2}, \theta=0\right)$ is performed. Equation (10) with these suggestions can be presented in the following form:

$$
g l / v^{2} \cdot\left(1-\frac{v^{2}}{V_{1}^{2}}\right) \bar{a}_{y}+\pi^{2} / 12\left(\frac{\bar{k}_{2} \cdot \bar{k}_{2}^{2}-\bar{k} 1 \cdot \bar{k}_{1}^{2}}{\overline{k_{1}} \cdot \overline{k_{1}^{2}} \cdot \bar{k}_{2} \cdot \bar{k}_{2}^{2}}\right) \bar{a}_{y}^{3}=0,
$$

then with $v>V_{1}$ and $\bar{k}_{1} \cdot \kappa_{1}^{2}>\bar{k}_{2} \cdot \kappa_{2}^{2}$ there are three steady states there (one stable and two unstable) and with $v>V_{1}$ and $\bar{k}_{1} \cdot \kappa_{1}^{2}>\bar{k}_{2} \cdot \kappa_{2}^{2}$ - there is one there (unstable). If $\bar{k}_{1} \kappa_{1}^{2}<\bar{k}_{2} \kappa_{2}^{2}$, then with $v>V_{1}$ there are three steady states there (one unstable and a pair of stable ones) - the case of stable steady states pair appearing (i.e. with $\bar{k}_{1} \kappa_{1}^{2}>\bar{k}_{2} \kappa_{2}^{2}$ bifurcation multitude branches in critical velocity small neighbourhood $v>V_{1}$ are located below returning point and with $\bar{k}_{1} \kappa_{1}^{2}<\bar{k}_{2} \kappa_{2}^{2}$ above it).

This way, in system (2) semitrailer parameters only implicitly influence conditions of safe linear movement mode stability loss (included into dimensionless drift coefficients on tractor truck axels), as opposed to conditions (9). Elimination of such qualitative differences between systems (1) and (2) required nonlinear connection restoring (with precision of up to third smallness term) for sub systems tractor truck-semitrailer which appears as a transverse force in the joint point. In case of the suggested approximate system (2) correction, an analytical means of building handling and folding curves reveals broadening of their usage area according to the normal acceleration parameter.

\section{Conclusions}

A general equation of handling and folding for a two-link road train nonlinear model is received, the handling equation co-insides in dimensionless form with the handling equation of the given tractor truck and can be used for evaluating conditions of divergent stability loss in an explicit form; 
obtained analytical conditions of safe linear movement mode stability loss are compared to the results of numerical building of road train nonlinear source model divergent stability loss diagram based on the parameter continuation method.

\section{References}

[1] Sedran, S. A truck dynamics model for driving simulators. Göteborg, Sweden 2016, p. 99.

[2] Zofka, M. R., Zöllner, J. M., Ren, M., Klemm, S., Kuhnt, F., Schamm, T., Marius, J. Z. Testing and validating high level components for automated driving: simulation framework for traffic scenarios testing and validating high level components for automated driving: simulation framework for traffic scenarios. 2016.

[3] Vo-Duy Thanh, C Ta Minh. A universal dynamic and kinematic model of vehicles. In Vehicle Power and Propulsion Conference (VPPC), 2015 IEEE, pages 1-6.

[4] Verbitskii, V. G., Bezverhyi, A. I., Tatievskyi, D. N. Handling and Stability Analysis of Vehicle Plane Motion. Mathematics and Computer Science. Available at: http://www.sciencepublishinggroup.com/journal/paperinfo?jo urnalid $=247 \&$ doi $=10.11648 /$ j.mcs.20180301.13.

[5] Gillespie, Thomas D. Fundamentals of Vehicle Dynamics. Society of Automotive Engineers, Inc. 1992, p. 470.

[6] Verbitskii, V. G., Lobas, L. G. Bifurcation of steady states for driven links of wheeled vehicles. Applied Mechanics, 1987, № 9, pp. 110-116.

[7] Arnold, V. I. Catastrophe Theory. Moscow: Nauka, 1990, p. 128.

[8] Bruce, J., Giblin, P. Curves and Singularities. Moscow: Mir, 1988, p. 262.

[9] Dhooge, A., Govaerts, W., Kuznetsov, Yu. A. Matcont: a matlab package fornumerical bifurcation analysis of ODEs, ACM Trans. Math. Software, 2002, № 29, pp. 141-164.

[10] Troger, H., Zeman, K. A nonlinear analysis of the generic types of loss of stability of the steady state motion of the tractor semitrailer. Vehicle System Dynamics, 1984, vol. 13, № 4, pp.
$161-172$.

[11] Fabio, Della Rossaa, Giampiero, Mastinub, Carlo, Piccardia. Bifurcation analysis of an automobile model negotiating a curve. Vehicle System Dynamics, 2012, vol. 50, № 10, pp. 1539-1562.

[12] Shinohara, Y. A geometric method for the numerical solution of non-linear equations and its application to non-linear oscillations. Publ. Res. Inst. Math. Sci., Kyoto Univ. 8, 1, 1972, pp. 13-42.

[13] Holodniok, M., Klic, A., Kubicek, M., Marek, M. Methods of Analysing Non-linear Dynamic Systems. Moscow: Mir, 1991, p. 368.

[14] Bautin, N. N. Behaviour of Dynamical Systems near the Boundery of the Stability Domain. Moscow: Nauka, 1984.

[15] Verbitskii, V. G., Lobas, L. G. Effect of external mechanical loads on the steady motions of wheeled machines. Applied Mechanics, 1990, № 7, pp. 97-102.

[16] Verbitskii, V. G., Lobas, L. G. Bifurcation of steady states of an assemblage of absolutely rigid and rolling elastic bodies. Izv. ASUSSR, MTT, 1991, № 3, pp. 30-37.

[17] Verbitskii, V. G., Lobas, L. G. Bifurcations of steady states in systems with rolling under constant force perturbations. Applied Mathematics and Mechanics, 1994, № 5, pp. 165-170.

[18] Pauwelussen, J. P. Analysis and prevention of excessive lateral behaviour of articulated vehicles. XII International Heavy Truck Conference, 13-15 September 1995, Budapest, Hungary.

[19] Ellis, J. R. Vehicle Dynamics. Moscow: Mashinostroenie, 1975, pp. 216 (Russian translation).

[20] Kravchenko, A. P., Verbitskii, V. G. Regarding the question of analyzing two-link road train handling. Multi transport systems: materials of VII All Russ. NTK, Krasnoyarsk, November 25-27. Novosibirsk: SGUPS publishing house, 2010, pp. 358-363.

[21] Lyapunov, A. M. Collected Papers in 6 Volumes, Vol.2. Izd. Akad. Nauk SSSR, Moskow, 1956.

[22] Verbitskii, V. G., Lobas, L. G. Real bifurcations of two-unit systems with rolling. Journal of Applied Mathematics and Mechanics, 1996, № 3, pp. 418-425. 